\title{
Importance of the Surface Electronic Structure in Heteroepitaxy
}

\author{
H. Wormeester \\ University of Twente, P.O. Box 217, 7500 AE Enschede, The Netherlands \\ E. Hüger \\ Physikalisches Institut, Technische Universität Clausthal, D-38678 Clausthal-Zellerfeld, Germany \\ E. Bauer \\ Arizona State University, Tempe, Arizona 85287
}

(Received 17 February 1998)

\begin{abstract}
Thin $\mathrm{Au}$ and $\mathrm{Pd}$ films were deposited on a $\mathrm{Nb}(001)$ surface covered with a monolayer (ML) of the respective metal and on a S-terminated $\mathrm{Nb}$. Although these ML's have the same lateral periodicity as the substrate, the films grown on them have a different structure. Films deposited on the metalterminated $\mathrm{Nb}$ surface have a structure determined by the geometric misfit between substrate and deposit (Au bcc, Pd hcp) while all films grown on the S-terminated $\mathrm{Nb}$ surface are fcc with (111) orientation. Photoelectron spectroscopy shows that $\mathrm{S}$ changes the electron density of states at the surface in such a way that the bonding of the metal is weakened, resulting in nonwetting. [S0031-9007(98)06689-7]
\end{abstract}

PACS numbers: $68.55 .-\mathrm{a}, 73.90 .+\mathrm{f}, 81.10 . \mathrm{Aj}$

The growth mode of thin films is determined by the surface free energies of film and substrate and by the free energy of the interface between them. On the basis of free energy considerations three growth modes, Frankvan der Merwe (FM), Volmer-Weber (VW), and StranskiKrastanov (SK), have been identified [1]. If the misfit between substrate and film is not zero, the film will grow in the SK mode, that is, initially monolayer by monolayer (ML by ML), followed by three-dimensional crystal growth, even if the surface free energies favor the FM mode, that is, ML by ML. However, even if the misfit is zero, growth in the FM mode frequently does not occur because the interface energy not only has elastic but also electronic contributions. In this Letter, we study the influence of the surface electronic structure on the growth mode of thin metal films. This is done by growing thin $\mathrm{Au}$ and $\mathrm{Pd}$ films on a $\mathrm{Nb}(001)$ substrate whose topmost layer is a pseudomorphic monolayer of the respective metals or of $\mathrm{S}$, which has almost the same atomic diameter as $\mathrm{Nb}$. The absence of a reconstruction on these surfaces was confirmed by very intense, low background $(1 \times$ 1) reflection high-energy electron diffraction (RHEED) patterns, with lattice parameters equal to that of the clean $\mathrm{Nb}(001)$ surface. The different overlayers provide substrates with the same lateral periodicity, but very different electronic structure.

The heteroepitaxial growth on the $\mathrm{Nb}(001)$ substrate was monitored with RHEED, Auger-electron spectroscopy (AES), and ultraviolet photoemission spectroscopy (UPS). The UHV system used has been described elsewhere [2]. It was also equipped with an $\mathrm{x}$-ray source, which allowed structural characterization of the films with core-level $\mathrm{x}$-ray photoelectron diffraction (XPD) technique. The evaporators were homebuilt and were operated at a deposition rate $0.001-0.01 \mathrm{ML} / \mathrm{s}$.
During deposition the pressure remained in the $10^{-8}$ $\mathrm{Pa}$ range. The experiments were performed with the substrate at a temperature of both 150 and $300 \mathrm{~K}$, with similar results at both temperatures. The measurements presented in this Letter were done at $150 \mathrm{~K}$ for $\mathrm{Pd}$ and $300 \mathrm{~K}$ for $\mathrm{Au}$. The $\mathrm{Nb}(001)$ crystal was prepared before the growth experiments in such a way that the outermost layer of the substrate is either $\mathrm{Au}, \mathrm{Pd}$, or S [3]. The normal emission UPS spectra of the substrates are shown in Fig. 1. The sputtered clean $\mathrm{Nb}$ surface has a high intensity but no pronounced features in this energy range. The S-terminated surface shows two prominent features, one at the Fermi edge, the other at $-6.3 \mathrm{eV}$ with a low intensity in between except for a weak feature at $-3.5 \mathrm{eV}$ that can probably be attributed to emission from the $\Delta_{1}$ $\mathrm{Nb}$ band [4]. The two strong emission features seen on the S-terminated $\mathrm{Nb}$ surface can be ascribed to the splitting of the $\mathrm{S} p$-orbital, i.e., $p_{x}, p_{y}$ at the higher binding energy and $p_{z}$ for emission near the Fermi edge. Such a splitting was also observed for the $\mathrm{S}$ covered $\mathrm{W}(001)$ surface, with features at -6 and $-1.4 \mathrm{eV}$ [5]. From electron emission angle dependent measurements (not shown) we found that both bands split with increasing $k_{\|}$, with a maximum splitting of $-1.4 \mathrm{eV}$ (Fermi edge feature) and $-2.3 \mathrm{eV}(-6.3 \mathrm{eV}$ feature) at the edge of the surface Brillouin zone. The prominent features of the substrate surfaces terminated by $1 \mathrm{ML} \mathrm{Au}$ and Pd lie between those of the $S$ covered surface. This indicates that the electronic structure of these surfaces and thus the bonding with a deposited film will be of a very different nature. For Pd [2] and Au [6], the features are similar to those observed on the $1 \mathrm{ML}$ covered $\mathrm{W}(001)$ surface, although they are much more intense and pronounced on the pseudomorphically covered $\mathrm{Nb}(001)$ surface. In the following we will characterize the metal-terminated 


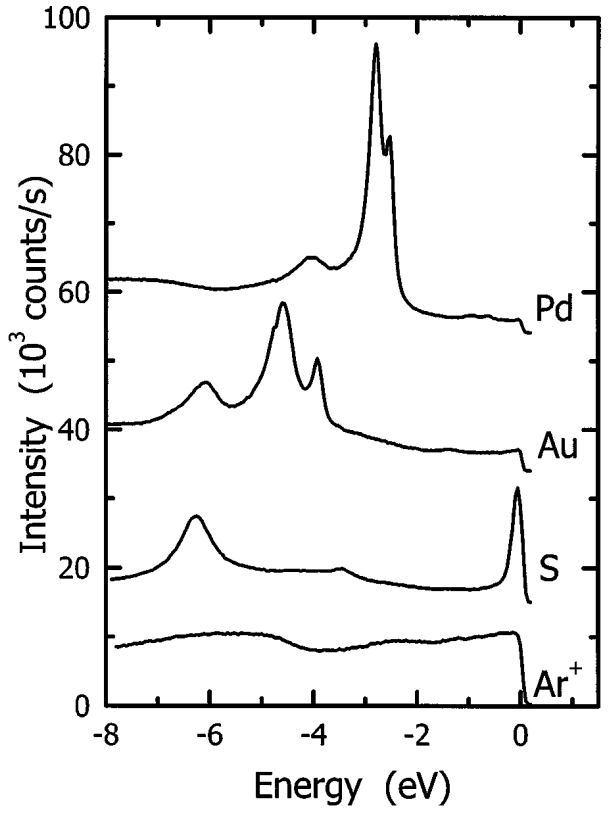

FIG. 1. Normal emission He-I excited UPS spectra of the mildly Ar sputtered $\mathrm{Nb}(001)$ surface and of $\mathrm{Nb}(001)$ surfaces terminated with $1 \mathrm{ML}$ of $\mathrm{S}, \mathrm{Au}$, and $\mathrm{Pd}$.

substrate surfaces by $\mathrm{Nb}+M, M$ indicating that the surface layer of the substrate is Au or Pd. These surfaces will be used as the starting substrate surfaces for the film growth.

Although AES is not a straightforward method for characterization of the growth mode, it can be used to show differences in growth mode. For $\mathrm{Pd} / \mathrm{W}(001)$, we found no change in slope up to $2.4 \mathrm{ML}$, at which point a small increase in slope was found [2]. This behavior was explained with the particular hcp growth mode and electron forward scattering. The same growth mode was found with UPS, RHEED, and XPD measurements for Pd deposited on the Pd-terminated surface, and on a lightly sputtered clean $\mathrm{Nb}$ surface. Because of the similar experimental results for the lightly sputtered clean $\mathrm{Nb}$ surface, we believe that the defects present on this surface have only minor influence on the growth mode compared to the influence of a $\mathrm{S}$ coverage. Because the Auger data of a $\mathrm{Pd}$ terminated $\mathrm{Nb}$ surface have an offset in the coverage scale, we present the data of the deposition on the sputtered $\mathrm{Nb}$ surface in Fig. 2. These have the same start signal as those of the $\mathrm{Nb}+\mathrm{S}$ substrate. The growth of $\mathrm{Pd}$ on $\mathrm{Nb}+\mathrm{S}$ is quite different from that on the sputtered $\mathrm{Nb}$ surface. The initial slope of the $\mathrm{Pd}$ AES signal is smaller than on the sputtered $\mathrm{Nb}$ surface and decreases suddenly at $4 \mathrm{ML}$. The Pd AES signal on the sputtered $\mathrm{Nb}$ surface shows the same linear increase with coverage up to $4 \mathrm{ML}$ as observed for $\mathrm{Pd} / \mathrm{W}(001)$ [2]. The $\mathrm{Nb}$ AES signals show a corresponding behavior. A different growth mode of the two films is obvious from the much smaller Pd signal on the $\mathrm{Nb}+\mathrm{S}$ surface (larger

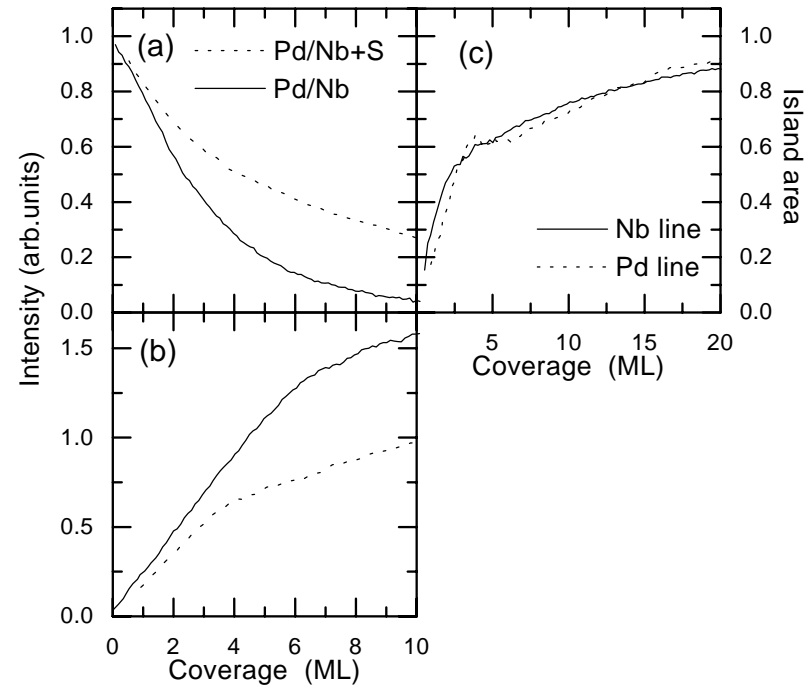

FIG. 2. Normal emission derivative Auger signals recorded during the growth of Pd films on the sputtered clean $\mathrm{Nb}(001)$ surface (solid curve) and on the S-terminated surface (dashed curve). (a) Normalized intensity of the $\mathrm{Nb}$ AES signal; (b) intensity of the Pd AES signal; (c) area covered by the $\mathrm{Pd}$ islands as determined from the $\mathrm{Nb}$ and Pd AES signal. Substrate temperature is $150 \mathrm{~K}$.

$\mathrm{Nb}$ signal) which indicates that the film does not grow ML by ML and that it is still not continuous at $10 \mathrm{ML}$. The fraction of the surface covered by Pd can be estimated from the evolution of the $\mathrm{Nb}$ and $\mathrm{Pd}$ Auger signals assuming that $\mathrm{Pd}$ grows in flat islands separated by bare surface regions. With inelastic mean free paths of $7 \AA$ for $\mathrm{Nb}$ and $9 \AA$ for Pd the covered surface fraction changes as shown in Fig. 2c. The value of the Pd saturation signal was chosen in such a manner that the absolute values of the two curves agree on the average.

The difference in growth mode is caused by fundamental differences in the electronic structure of the deposit as illustrated by the UPS spectra taken during growth on the various substrate surfaces (Fig. 3). While the UPS spectra of $\mathrm{Pd}$ on $\mathrm{Nb}+\mathrm{Pd}$ are like those of $\mathrm{Pd}$ on $\mathrm{W}(001)$ [2], characteristic of the growth of hcp (112̄0) oriented $\mathrm{Pd}$, the growth of $\mathrm{Pd}$ on $\mathrm{Nb}+\mathrm{S}$ leads to UPS spectra comparable to those of Pd on W(110) [7] beyond the second monolayer, characteristic for (111) oriented fcc Pd. The UPS spectra of Au also show the growth of a (111) oriented fcc film on the $\mathrm{Nb}+\mathrm{S}$ surface, similar to the growth of $\mathrm{Au}$ [8] on W(110) beyond the second ML. On the metal-terminated substrate surface, the UPS spectra resemble those of bcc Au [6]. The orientation of the films deposited on the metal-terminated surfaces was also determined with XPD and transmission RHEED patterns. $\mathrm{Au}$ was found to be bcc with the orientation relationship $(001)_{f}\left\|(001)_{s},[100]_{f}\right\|[110]_{s}$, Pd hcp with the orientation relationship $(11 \overline{2} 0)_{f}\left\|(001)_{s},[0001]_{f}\right\|[110]_{s}$, in complete analogy to the growth on $\mathrm{W}(001)$ reported previously and explained by misfit considerations $[2,9]$. 


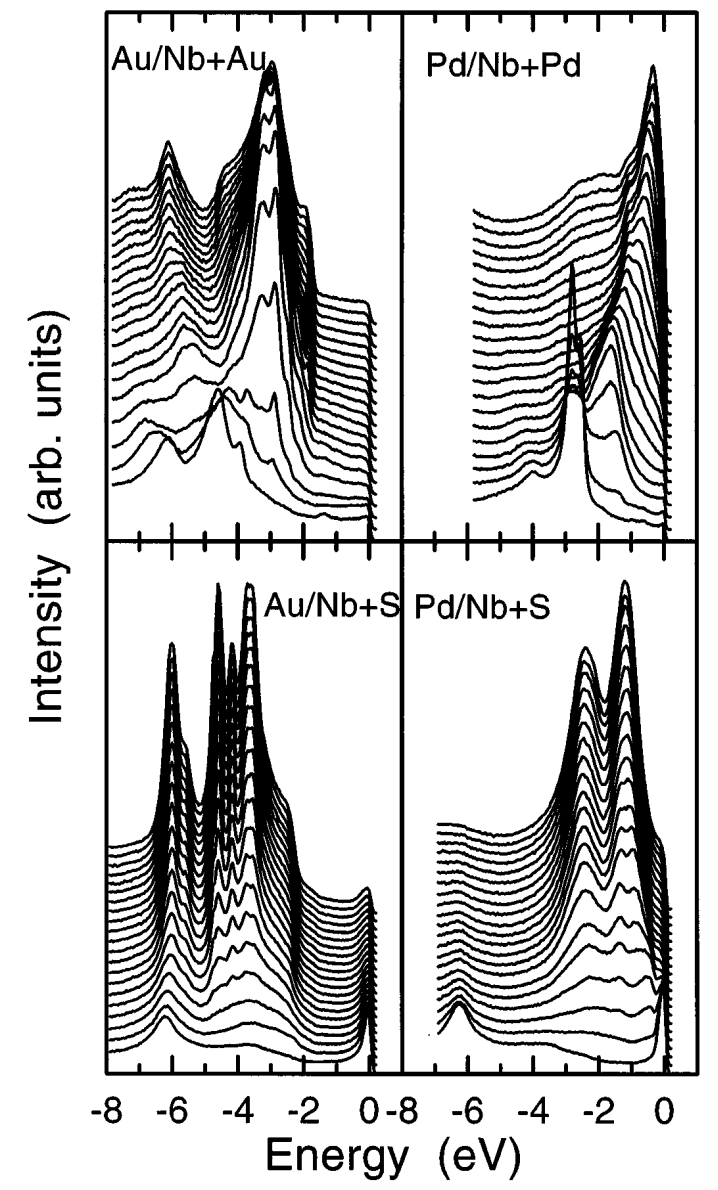

FIG. 3. Normal emission He-I excited UPS spectra recorded during the growth of $\mathrm{Au}(300 \mathrm{~K})$ and $\mathrm{Pd}(150 \mathrm{~K})$ films on the $\mathrm{Nb}(001)$ surface covered with $1 \mathrm{ML}$ of $\mathrm{Au}$ and $\mathrm{Pd}$, respectively (upper row), and with $1 \mathrm{ML}$ of $\mathrm{S}$ (lower row). The lowest spectrum in every series represents the starting situation, while the topmost spectrum of every series represents: $22.5 \mathrm{ML}$ $\mathrm{Au} / \mathrm{Nb}+\mathrm{Au} ; 6.2 \mathrm{ML} \mathrm{Pd} / \mathrm{Nb}+\mathrm{Pd} ; 13.6 \mathrm{ML} \mathrm{Au} / \mathrm{Nb}+\mathrm{S}$; and $11.3 \mathrm{ML} \mathrm{Pd} / \mathrm{Nb}+\mathrm{S}$.

These measurements show that the electronic structure of the substrate has a profound influence on the growth mode as the lattice constant of the $\mathrm{S}$ and metal-terminated $\mathrm{Nb}$ surface is the same. In AES measurements, we observed that the decrease of the S AES signal upon deposition was equal to the decrease of the intensity of the $\mathrm{Nb}$ AES line. This shows that the $\mathrm{S}$ remains at the interface during the growth of the metal layer. Oxygen on the $\mathrm{Nb}(001)$ surface influences the growth mode in the same manner as S. Already $10 \%$ of $\mathrm{S}$ on the $\mathrm{Nb}$ surface or $25 \%$ oxygen was found to be enough for the growth of a (111) oriented fcc film. However, these measurements are not considered here as certainly oxygen is known to induce various coverage-dependent reconstructions, so that the geometrical starting conditions compared with the metal-terminated surfaces were not equal.

The evolution of the film structure during growth was also studied with RHEED. For $\mathrm{Au}$ on $\mathrm{Nb}+\mathrm{Au}$, the in- tensity of the initial $(1 \times 1)$ pattern decreased rapidly, and the background increased. On the other hand, during growth on the $\mathrm{Nb}+\mathrm{S}$ surface, spot intensity and background did not change up to almost $1 \mathrm{ML}$. This indicates that the $\mathrm{Au}$ islands on the $\mathrm{Nb}+\mathrm{S}$ surface must have a distance equal to or larger than the transfer width of the RHEED system, which is estimated to be $35 \AA$ parallel to the shadow edge [6]. Above $1 \mathrm{ML}$ diffuse rings become visible (Fig. 4a), which sharpen with increasing coverage and can unequivocally be attributed to fcc Au. These rings show that there is no specific orientation relation between substrate and film. Streaks hardly visible at 1.7 ML become more intense with increasing coverage, while the polycrystalline rings weaken and can no longer be observed beyond $5 \mathrm{ML}$. Two different set of streaks are visible in Fig. 4a representing a periodicity of $d_{\mathrm{Au}}$ and $\sqrt{3 / 4} d_{\mathrm{Au}}$, with $d_{\mathrm{Au}}$ the nearest neighbor distance, corresponding to the $\mathrm{Au}\langle 110\rangle$ and $\langle 211\rangle$ directions, characteristic for (111) oriented Au. Rotation of the sample azimuth does not change the relative intensity of the $\langle 110\rangle$ and $\langle 211\rangle$ streaks which shows that the (111) orientation is not epitaxial but a fiber texture. The evolution of such a fiber texture during coalescence of individual crystals into a continuous film has been known for a long time from the growth of metals on UHV-cleaved ionic crystals (see, for example, Ref. [10]).

Figure 1 shows that the S-terminated surface layer is characterized by two strong emission features from the $3 p$ orbitals, one at the Fermi edge and one at $6.2 \mathrm{eV}$ binding energy. Au and Pd terminated surfaces have features in (a)
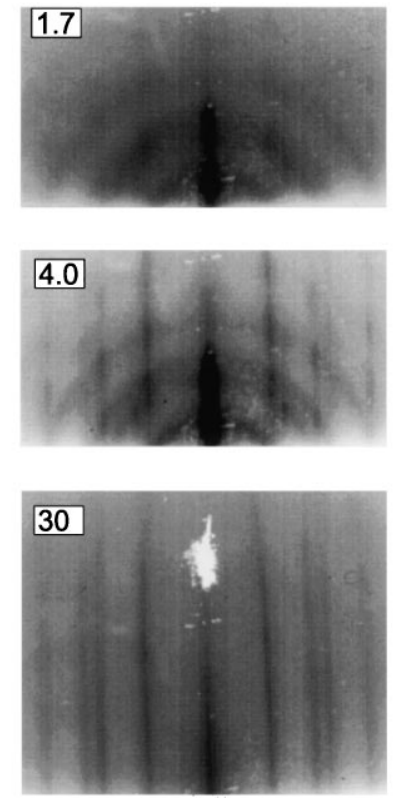

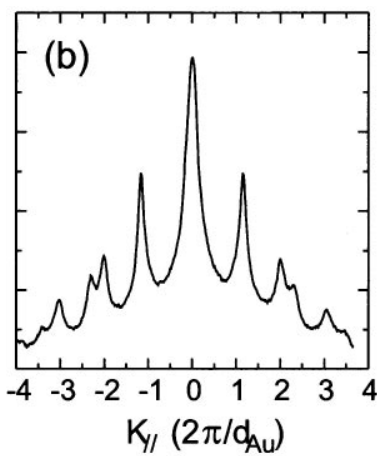

$K_{/ /}\left(2 \pi / d_{A u}\right)$
FIG. 4. RHEED patterns of $\mathrm{Au} / \mathrm{Nb}+\mathrm{S}$. (a) $1.7 \mathrm{ML} \mathrm{Au}$ showing diffuse polycrystalline rings, $4 \mathrm{ML} \mathrm{Au}$, showing polycrystalline rings and streaks, and $30 \mathrm{ML}$, showing sharp streaks. (b) Line scan parallel to the shadow edge of the $30 \mathrm{ML}$ $\mathrm{Au}$ RHEED pattern. Substrate temperature is $300 \mathrm{~K}$. 
the -2 to $-6 \mathrm{eV}$ region (Fig. 1). Bonding between the $\mathrm{S}$-terminated surface and the $\mathrm{Au}$ or Pd deposit is thus difficult as the electron density of states do not overlap, at least not at the $\Gamma$ point. The hcp Pd layer has a high density of states at the Fermi edge as the S-terminated surface does so that bonding with this surface appears possible. However, this high density of states develops only after a few pseudomorphic (bcc) layers have been deposited. The density of states of the pseudomorphic Pd layers is concentrated at binding energies above $1 \mathrm{eV}$ so that there is very little overlap in the critical initial growth phase. The bonding difference in the initial phase is also observed in the development of the UPS spectra during growth (Fig. 3). The decrease of the $-6.2 \mathrm{eV}$ feature and the Fermi-edge feature on the S-terminated surface with increasing coverage of $\mathrm{Pd}$ and $\mathrm{Au}$, respectively, shows that these features do not mix with the valence electrons of the metal deposited on the $\mathrm{Nb}+\mathrm{S}$ surface. Although the spectra of the thin films deposited on the S-terminated surface change in the initial growth stage, this is mainly due to a change in intensity of the fcc (111) related features of the deposit. These features remain at the same energy. The contrary is the case for deposition on the metal-terminated $\mathrm{Nb}$ surfaces. In the initial phase, the valence band of the deposit changes dramatically as indicated by the change in width and energy position of the prominent features during the deposition of the first several monolayers. The Au-terminated surface shows a feature at $-6 \mathrm{eV}$ that is also seen for the thick layer, but these features are of a very different origin. This feature shifts first to higher binding energy while later it evolves from a feature that is first observed around $-5 \mathrm{eV}$. In $\mathrm{Pd}$ layers an evolution of the energy position and width of the electron emission towards the Fermi energy is observed.

The influence of the $\mathrm{S}$ (and $\mathrm{O}$ ) is of a thermodynamic nature, and not of kinetic origin. The change of diffusion properties, like a Ehrlich-Schwoebel barrier, etc., would change the morphology of islands and the growing film, i.e., compact or fractal islands, smooth or rough films. In this work also the crystal structure of the film is completely changed. This is no longer the result of a change in diffusion barriers as also the geometric positions of the atoms in the submonolayer are affected and no pseudomorphic layer is grown. This effect is attributed to the much weaker bond between adsorbate and substrate. The wetting of a substrate by a thin film depends on the surface and interface free energies of the materials involved. Considering the termination layer as part of the $\mathrm{Nb}$ substrate our observations show that S dramatically changes the electronic contribution to its surface or interface free energy. The electronic component of the free energy determines the growth mode dramatically already from the very beginning of the growth process. In contrast, the elastic component which is determined by the misfit between substrate and film becomes apparent only after the deposition of several monolayers when the misfit is sufficiently small [as is the case for bcc $\mathrm{Au}$ on $\mathrm{Nb}(001)$ ]. The effect of $\mathrm{S}$ cannot be compared with that of a surfactant [11] because a surfactant induces layer-by-layer growth and floats on top of the growing film. S is rather an "anti-interfactant" as it remains at the interface and suppresses two-dimensional growth in contrast to an interfactant.

In summary, we found that the growth of $\mathrm{Au}$ and $\mathrm{Pd}$ on a metal-terminated $\mathrm{Nb}(1 \times 1)$ surface is very different from that on a S-terminated $\mathrm{Nb}(1 \times 1)$ surface. In the first case the thin metal films grow epitaxially in hcp or bcc structure, and in the second case they grow initially polycrystalline with fcc structure and develop a strong preferred (111) fiber texture during coalescence. The same behavior was also observed for $\mathrm{Cu}$, i.e., a $(11 \overline{2} 0)$ oriented hcp film on $\mathrm{Cu}$ terminated $\mathrm{Nb}(001)$ and a (111) oriented fcc film for S-terminated $\mathrm{Nb}(001)$. Epitaxy occurs in the case of strong film-substrate interaction in which misfit considerations determine the orientation, while the fiber texture develops when these interactions are weak and, therefore, also the azimuthal variation of the interfacial energy. The difference in growth is a consequence of the different electronic structure which determines bonding between deposit and substrate.

This work was supported by the Deutsche Forschungsgemeinschaft. One of the authors (H.W.) acknowledges support from the Alexander von Humboldt Foundation and the Royal Netherlands Academy of Sciences (KNAW).

[1] E. Bauer, Z. Kristallogr. 110, 372 (1958).

[2] H. Wormeester, E. Hüger, and E. Bauer, Phys. Rev. B 54, 17108 (1996).

[3] E. Hüger, H. Wormeester, and E. Bauer (unpublished).

[4] B.-S. Fang, C. A. Ballentine, and J. L. Erskine, Phys. Rev. B 38, 4299 (1988).

[5] A. Bhattacharya, L. J. Clarke, and L. Morales de la Garza, J. Chem. Soc. Faraday Trans. 1 77, 2223 (1981).

[6] H. Wormeester, E. Hüger, and E. Bauer, Phys. Rev. B 57, 10120 (1998).

[7] H. Knoppe and E. Bauer, Z. Phys. Chem. 202, 45 (1997).

[8] H. Knoppe and E. Bauer, Phys. Rev. B 48, 5621 (1993).

[9] H. Wormeester, E. Hüger, and E. Bauer, Phys. Rev. Lett. 77, 1540 (1996).

[10] E. Bauer et al., in Basic Problems in Thin Film Physics, edited by R. Niedermayer and H. Mayer (Vanderhoek and Ruprecht, Göttingen, 1966), p. 135.

[11] For an extensive list of work published in the area of surfactants in MBE, see W.F. Egelhoff, Surf. Sci. (to be published). 